United Nations Educational Scientific and Cultural Organization and

International Atomic Energy Agency

THE ABDUS SALAM INTERNATIONAL CENTRE FOR THEORETICAL PHYSICS

\title{
CRITICAL EIGENVALUES FOR A NONLINEAR PROBLEM
}

Ould Ahmed Izid Bih Isselkou ${ }^{1}$

Faculté des Sciences et Techniques, B.P. 5026 Nouakchott, Mauritanie and

The Abdus Salam International Centre for Theoretical Physics, Trieste, Italy.

\begin{abstract}
In this work, we give the explicit solutions of the problem

$$
\left(P_{\lambda}\right)\left\{\begin{array}{l}
\Delta u+\lambda(1+u)=0 \text { in } B_{1}, \\
u>0 \text { in } B_{1} \\
u=0 \text { on } \partial B_{1}
\end{array}\right.
$$
\end{abstract}

where $B_{1}=\left\{x \in \Re^{3},\|x\|<1\right\}$, and $\lambda$ is a positive constant.

The supremum $\lambda^{\star}$ of values such that $\left(P_{\lambda}\right)$ admits a solution is equal to $\pi^{2}$; we show that the problem $\left(P_{\pi^{2}}\right)$ doesn't admit a solution (see Section 3).

We study the application

$$
\left.\lambda^{\star}:\right] 1, \infty\left[\longrightarrow \Re_{+},\right.
$$

where $\lambda^{\star}(\alpha)$ is the supremum of positive values such that the problem

$$
\left(P_{\lambda}^{\alpha}\right)\left\{\begin{array}{l}
\Delta u+\lambda(1+u)^{\alpha}=0 \text { in } B_{1}, \\
u>0 \text { in } B_{1}, \\
u=0 \text { on } \partial B_{1}
\end{array}\right.
$$

admits a solution. We show that $\lambda^{\star}$ is a decreasing function, with

$$
\lim _{\alpha \rightarrow \infty} \lambda^{\star}(\alpha)=0 \text { and } \lim _{\alpha \rightarrow \infty} u_{\lambda^{\star}(\alpha)}(0)=+\infty .
$$

where $u_{\lambda^{\star}(\alpha)}$ is the unique solution of the problem $\left(P_{\lambda^{\star}(\alpha)}^{\alpha}\right)$ (see Section 4). In the last section, we give a numerical approximation of $\lambda^{\star}(2)$.

$$
\text { MIRAMARE - TRIESTE }
$$

November 2000

\footnotetext{
${ }^{1}$ Regular Associate of the Abdus Salam ICTP. Member of the Network "PDE, Control and Modeling".
} 


\section{Introduction}

In this work, we deal with the following problem

$$
\left(P_{\lambda}^{\alpha}\right)\left\{\begin{array}{l}
\Delta u+\lambda(1+u)^{\alpha}=0 \text { in } B_{1}, \\
u>0 \text { in } B_{1}, \\
u=0 \text { on } \partial B_{1},
\end{array}\right.
$$

where $B_{1}$ is the open unit ball of $\Re^{n}, n \geq 3, \lambda>0$ and $\alpha \geq 1$.

The problem $\left(P_{\lambda}^{\alpha}\right)$ is a particular form of the following general one

$$
\left\{\begin{array}{l}
L u=-\Sigma_{i, j} \frac{\partial}{\partial x_{i}}\left(a_{i j}(x) \frac{\partial u(x)}{\partial x_{j}}\right)+c(x) u(x)=\lambda f(x, u) \text { in } \Omega \\
u=0 \text { on } \partial \Omega
\end{array}\right.
$$

where $\Omega$ is a regular bounded domain of $\Re^{n}, L$ is uniformly elliptic in $\Omega$ and $f(x, 0)>0$, for $x \in \Omega$.

The previous problem arises in many mathematical models (combustion, thermal explosions, nonlinear heat generation and gravitational equilibrium of polytropic stars, see [2] for detailed references).

It is known (see [8],[2],[1] and [5]), that for every $\alpha>1$, there exists a critical constant $\lambda^{\star}(\alpha)>0$, such that the problem $\left(P_{\lambda}^{\alpha}\right)$ admits two ordered solutions if $0<\lambda<\lambda^{\star}(\alpha)$, only one solution if $\lambda=\lambda^{\star}(\alpha)$ and no solution if $\lambda>\lambda^{\star}(\alpha)$.

Our aim is to get more details about the application

$$
\lambda^{\star}:[1, \infty[\rightarrow \Re+.
$$

We have already pointed out concrete situations where problem $\left(P_{\lambda}^{\alpha}\right)$ arises, let us add that if we know the exact value of $\lambda^{\star}(\alpha)$, we can show (for instance) that the problem

$$
\left\{\begin{array}{l}
\Delta u+a(x)(1+u)^{\alpha}=0 \text { in } \Omega, \\
u>0 \text { in } \Omega \\
u=0 \text { on } \partial \Omega
\end{array}\right.
$$

doesn't admit a solution, if $a(x)>\lambda^{\star}(\alpha)$ for every $x \in \bar{\Omega}$, and $a$ is regular enough on $\Omega$.

\section{Some Previous Results}

Let us consider the problem $\left(P_{\lambda}^{\alpha}\right)$ on $\Omega$ instead of $B_{1}, \alpha>1$. Let $u_{\lambda}$ be a solution of this problem. If we put $v_{\lambda}=\lambda^{\frac{1}{\alpha-1}} u_{\lambda}+\lambda^{\frac{1}{\alpha-1}}$, then $v_{\lambda}$ verifies

$$
\left\{\begin{array}{l}
\Delta v_{\lambda}+v_{\lambda}^{\alpha}=0 \text { in } \Omega \\
v_{\lambda}>0 \text { in } \Omega \\
v_{\lambda}=\lambda^{\frac{1}{\alpha-1}}=\epsilon \text { on } \partial \Omega
\end{array}\right.
$$

So one can study the previous Dirichlet's problem (with constant boundary data) to get existence results and critical boundary constants $\epsilon^{\star}(\Omega, \alpha)$, such that, if $\epsilon>\epsilon^{\star}(\Omega, \alpha)$, the previous problem doesn't admit a solution. One can study this problem with more general nonlinearities $f(x, u)$, 
on adequate domains. This was the method in [5].

Let us choose

$$
\Omega=B_{r}=\left\{x \in \Re^{n},\|x\|<r\right\}, r>0,
$$

and let $\lambda_{r}^{\star}(\alpha)$ be the supremum of values $\lambda$ for which the previous problem admits a solution, then we have

\section{Lemma 1}

$$
\lambda_{r}^{\star}(\alpha)=r^{-2} \lambda_{1}^{\star}(\alpha), \epsilon^{\star}\left(B_{r}, \alpha\right)=r^{\frac{2}{1-\alpha}} \epsilon^{\star}\left(B_{1}, \alpha\right)
$$

and

$$
\lambda_{1}^{\star}(\alpha)=\epsilon^{\star}\left(B_{1}, \alpha\right)^{\alpha-1} .
$$

Proof

See [5] or [6].

The previous lemma gives a reason to deal firstly with the case $\Omega=B_{1}$.

Let us recall the Sobolev critical exponent case,

Proposition 1 Consider the problem $\left(P_{\lambda}^{\alpha}\right)$, if

$$
\alpha=\frac{n+2}{n-2}, \text { then } \lambda^{\star}(\alpha)=\frac{n(n-2)}{4} .
$$

Proof

See Proposition 1 in [7].

Proposition 2 Every $C^{2}$-solution $u$ of $\left(P_{\lambda}^{\alpha}\right)$ is radially symmetrical with respect to the origin and decreasing.

Proof

See Theorem 1 in [3].

Proposition 3 For every $\lambda$ such that $0<\lambda \leq \lambda^{\star}(\alpha)$, let $u_{\lambda}$ be the smaller solution of $\left(P_{\lambda}^{\alpha}\right)$, then $u_{\lambda}$ is analytic.

Proof

We know from Proposition 2, that $u_{\lambda}$ is spherically symmetrical. We also know (see [9], Theorem 3.2 , p. 227) that

$$
u_{\lambda}(r)=\lim _{i \rightarrow \infty} u_{\lambda}^{(i)}(r), 0 \leq r \leq 1,
$$

where $u_{\lambda}^{(i)}$ is the solution of the linear problem

$$
\left(P_{\lambda, i}^{\alpha}\right)\left\{\begin{array}{l}
\Delta u_{\lambda}^{(i)}+\lambda\left(1+u_{\lambda}^{(i-1)}\right)^{\alpha}=0 \text { in } B_{1} \\
u_{\lambda}^{(i)}>0 \text { in } B_{1} \\
u_{\lambda}^{(i)}=0 \text { on } \partial B_{1}
\end{array}\right.
$$


with $u_{\lambda}^{(0)} \equiv 0$.

It is easy to verify that $u_{\lambda}^{(1)}$ is a radially symmetrical, decreasing analytic function of $r=\|x\|$, so are $u_{\lambda}^{(2)}$ and (by induction) $u_{\lambda}^{(i)}$ for every $i>1$ (see Theorem 1 ' in [3], p.221). As the sequence $u_{\lambda}^{(i)} \leq u_{\lambda}^{(i+1)}$, one can use Dini's Lemma to conclude that $u_{\lambda}$ is analytic.

\section{Explicit Solutions for $\alpha=1, n=3$.}

Let us consider the problem $\left(P_{\lambda}\right)$ again with $n=3$,

Proposition 4 For every $0<\lambda<\pi^{2}$, the problem $\left(P_{\lambda}\right)$ admits the unique radially symmetrical solution

$$
\begin{gathered}
u_{\lambda}(r)=\frac{\sqrt{\lambda}}{\sin (\sqrt{\lambda})} \frac{\sin r \sqrt{\lambda}}{r \sqrt{\lambda}}-1,0<r \leq 1, \text { and } u_{\lambda}(0)=\frac{\sqrt{\lambda}}{\sin (\sqrt{\lambda})}-1 . \\
\lim _{\lambda \rightarrow \pi^{2}} u_{\lambda}(r)=+\infty, \text { for every } 0 \leq r<1 .
\end{gathered}
$$

If $\lambda \geq \pi^{2}$, the problem $\left(P_{\lambda}\right)$ doesn't admit a solution.

Proof

Let $\lambda$ be a positive real such that the problem $\left(P_{\lambda}\right)$ admits a solution $u_{\lambda}$. The unicity of such a solution was proved in [8]. From Proposition 3 , we infer that $u_{\lambda}$ is radially symmetrical and analytic. So we can rewrite $\left(P_{\lambda}\right)$ as follows

$$
\left(P_{\lambda}^{\prime}\right)\left\{\begin{array}{l}
u_{\lambda} "(r)+\frac{2}{r} u_{\lambda}^{\prime}(r)+\lambda\left(1+u_{\lambda}(r)\right)=0 \quad \forall 0<r<1, \\
u_{\lambda}(0)=c_{0}, u_{\lambda}^{\prime}(0)=0, u_{\lambda}(1)=0 .
\end{array}\right.
$$

So we obtain that

$$
r\left(u_{\lambda} "(r)+\lambda\left(1+u_{\lambda}(r)\right)\right)=-2 u_{\lambda}^{\prime}(r), 0 \leq r<1 .
$$

If

$$
u_{\lambda}(r)=\Sigma_{j=0}^{\infty} c_{j} r^{j} \text { on }[0,1]
$$

we get that

$$
(j+1)(j) c_{j+1}+\lambda c_{j-1}=-2(j+1) c_{j+1}, \forall j \geq 2 \text { and } 2 c_{2}+\lambda\left(1+c_{0}\right)=-4 c_{2}
$$

From these equalities, we obtain

$$
c_{j}=-\frac{\lambda}{(j+1) j} c_{j-2}, \forall j \geq 3 \text { and } c_{2}=-\frac{\lambda\left(1+c_{0}\right)}{6} .
$$

As $c_{1}=u_{\lambda}^{\prime}(0)=0$, we infer that $c_{j}=0$ for every odd integer $j$ and

if $j$ is even

$$
c_{j}=\frac{(-\lambda)^{\frac{j-2}{2}} c_{2}}{(j+1)(j) \ldots(4)}=\frac{(-\lambda)^{\frac{j}{2}}\left(1+c_{0}\right)}{(j+1) !}
$$

Finally, we get

$$
u_{\lambda}(r)=\Sigma_{j=0}^{\infty} \frac{(-\lambda)^{j}\left(1+c_{0}\right)}{(2 j+1) !} r^{2 j}-1
$$


When $j=0, \frac{(-\lambda)^{j}\left(1+c_{0}\right)}{(2 j+1) !}=1+c_{0}$, that's why we have the term -1 , on the right, in the expression of $u_{\lambda}$.

We can rewrite $u_{\lambda}$ as follows

$$
u_{\lambda}(r)=\frac{\left(1+c_{0}\right)}{r \sqrt{\lambda}} \Sigma_{j=0}^{\infty} \frac{(-1)^{j}(r \sqrt{\lambda})^{2 j+1}}{(2 j+1) !}-1 .
$$

We can identify the well-known Taylor's series of the function $\sin (r \sqrt{\lambda})$ and get

$$
u_{\lambda}(r)=\frac{\left(1+c_{0}\right)}{r \sqrt{\lambda}} \sin (r \sqrt{\lambda})-1 ; u_{\lambda}(1)=0=\frac{\left(1+c_{0}\right)}{\sqrt{\lambda}} \sin (\sqrt{\lambda})-1 .
$$

We infer that

$$
c_{0}=\frac{\sqrt{\lambda}-\sin (\sqrt{\lambda})}{\sin (\sqrt{\lambda})}=\frac{\sqrt{\lambda}}{\sin (\sqrt{\lambda})}-1
$$

Substituting in the expression of $u_{\lambda}$, we obtain

$$
u_{\lambda}(r)=\frac{1}{r \sin (\sqrt{\lambda})} \sin (r \sqrt{\lambda})-1=\frac{\sqrt{\lambda}}{\sin \sqrt{\lambda}} \frac{\sin (r \sqrt{\lambda})}{r \sqrt{\lambda}}-1 .
$$

As $u_{\lambda}(r) \geq 0$ on $[0,1]$, one can verify that necessarily $\lambda<\pi^{2}$.

Let us consider the function

$$
\left\{\begin{array}{l}
g:[0, \pi] \rightarrow \Re \\
g(x)=\frac{\sin x}{x}, \text { if } x \neq 0, g(0)=1 .
\end{array}\right.
$$

On ]0, $\pi$, the function $g$ is $C^{\infty}$ and verifies $g^{\prime}(x)=\frac{x \cos x-\sin x}{x^{2}}$. As $(x \cos x-\sin x)^{\prime}=-x \sin x \leq 0$, on $\left.] 0, \pi\right]$, it is straightfoward to verify that $g$ is a decreasing function on $[0, \pi]$, with $g(\pi)=0$. As

$$
u_{\lambda}(r)=\frac{\sqrt{\lambda}}{\sin \sqrt{\lambda}} \frac{\sin (r \sqrt{\lambda})}{r \sqrt{\lambda}}-1=\frac{\frac{\sin (r \sqrt{\lambda})}{r \sqrt{\lambda}}}{\frac{\sin (\sqrt{\lambda})}{\sqrt{\lambda}}}-1=\frac{g(r \sqrt{\lambda})}{g(\sqrt{\lambda})}-1 \text { and } r \sqrt{\lambda} \leq \sqrt{\lambda}
$$

we infer that

$$
\forall 0<\lambda<\pi^{2}, u_{\lambda}(r)=\frac{g(r \sqrt{\lambda})}{g(\sqrt{\lambda})}-1 \geq 0, \forall 0 \leq r \leq 1 .
$$

It is immediate to verify that

$$
\lim _{\lambda \rightarrow \pi ; \lambda<\pi} u_{\lambda}(r)=+\infty, \forall 0 \leq r<1 .
$$

We conclude that $\lambda^{\star}(1)=\pi^{2}$ and there is no solution for the problem $\left(P_{\lambda^{\star}(1)}\right)$.

Remark 1 The problem $\left(P_{\lambda^{\star}(1)}\right)$ doesn't admit a solution, whereas it is known that the problem $\left(P_{\lambda^{\star}}(\alpha)\right)$ admits a unique solution, if $\alpha>1$. 


\section{Some Properties of the Application $\lambda^{\star}$}

Let $u_{\lambda^{\star}(\alpha)}$ be the unique solution of the problem $\left(P_{\lambda^{\star}(\alpha)}^{\alpha}\right)$, when $\alpha>1$.

Proposition 5 The application

$$
\left.\lambda^{\star}:\right] 1, \infty\left[\longrightarrow \Re_{+},\right.
$$

is decreasing and verifies

$$
\lim _{\alpha \rightarrow \infty} \lambda^{\star}(\alpha)=0 \text { and } \lim _{\alpha \rightarrow \infty} u_{\lambda^{\star}(\alpha)}(0)=+\infty
$$

Proof

Let $1<\alpha_{1}<\alpha_{2}$ and $u_{\lambda^{\star}\left(\alpha_{2}\right)}$ be the unique solution of

$$
\left\{\begin{array}{l}
\Delta u+\lambda^{\star}\left(\alpha_{2}\right)(1+u)^{\alpha_{2}}=0 \text { in } B_{1}, \\
u>0 \text { in } B_{1} \\
u=0 \text { on } \partial B_{1} .
\end{array}\right.
$$

As $\alpha_{2}>\alpha_{1}$, using the Maximum Principle, we get that

$$
0=\Delta u_{\lambda^{\star}\left(\alpha_{2}\right)}+\lambda^{\star}\left(\alpha_{2}\right)\left(1+u_{\lambda^{\star}\left(\alpha_{2}\right)}\right)^{\alpha_{2}}>\Delta u_{\lambda^{\star}\left(\alpha_{2}\right)}+\lambda^{\star}\left(\alpha_{2}\right)\left(1+u_{\lambda^{\star}\left(\alpha_{2}\right)}\right)^{\alpha_{1}} \text { in } B_{1} \text {. }
$$

So we infer that $u_{\lambda^{\star}\left(\alpha_{2}\right)}$ is a supersolution of the problem

$$
\left\{\begin{array}{l}
\Delta v+\lambda^{\star}\left(\alpha_{2}\right)(1+v)^{\alpha_{1}}=0 \text { in } B_{1} \\
v>0 \text { in } B_{1} \\
v=0 \text { on } \partial B_{1}
\end{array}\right.
$$

We can choose the function $\phi \equiv 0$ as a subsolution for the previous problem and obtain (by Nagumo's Theorem) a solution of this problem. Using the maximality of $\lambda^{\star}\left(\alpha_{1}\right)$ and the uniqueness of $u_{\alpha_{1}}$, we infer that

$$
\lambda^{\star}\left(\alpha_{2}\right)<\lambda^{\star}\left(\alpha_{1}\right)
$$

As we have $\lambda^{\star}\left(\alpha_{2}\right)<\lambda^{\star}\left(\alpha_{1}\right)$, we know that there exists a "small" solution $w$ such that

$$
\left\{\begin{array}{l}
\Delta w+\lambda^{\star}\left(\alpha_{2}\right)(1+w)^{\alpha_{1}}=0 \text { in } B_{1}, \\
w>0 \text { in } B_{1} \\
w=0 \text { on } \partial B_{1} .
\end{array}\right.
$$

We have already mentioned that for every $\lambda$, the "small" solution, when it exists, verifies

$$
u_{\lambda}(r)=\lim _{j \rightarrow \infty} u_{\lambda}^{(j)}(r)
$$

where $u_{\lambda}^{(0)} \equiv 0$ and $u_{\lambda}^{(j)}$ is the solution of the linear problem $\left(P_{\lambda, j}^{\alpha}\right)$. Let us show that $u_{\lambda^{\star}\left(\alpha_{2}\right)} \geq w$, on $B_{1}$.

$$
\left\{\begin{array}{l}
\Delta\left(u_{\lambda^{\star}\left(\alpha_{2}\right)}^{(1)}-w\right)=-\lambda^{\star}\left(\alpha_{2}\right)\left((1+w)^{\alpha_{2}}-1\right) \leq 0 \text { in } B_{1}, \\
u_{\lambda^{\star}\left(\alpha_{2}\right)}^{(1)}-w=0 \text { on } \partial B_{1} .
\end{array}\right.
$$


The Maximum Principle implies that

$$
u_{\lambda^{\star}\left(\alpha_{2}\right)}^{(1)} \geq w, \text { on } B_{1} \text {. }
$$

One can reiterate the previous argument to show that

$$
u_{\lambda^{\star}\left(\alpha_{2}\right)}^{(j)} \geq w, \text { on } B_{1}, \forall j \geq 1
$$

so we deduce that

$$
u_{\lambda^{\star}\left(\alpha_{2}\right)} \geq w, \text { on } B_{1}, \forall \alpha_{2} \geq \alpha_{1}
$$

As the function $\lambda^{\star}$ is a positive decreasing function on $] 1, \infty[$, there are only two possible cases

$$
\begin{cases}(i) & \lim _{\alpha \rightarrow \infty} \lambda^{\star}(\alpha)=0 \\ \text { or } & \\ (i i) & \lim _{\alpha \rightarrow \infty} \lambda^{\star}(\alpha)=l>0 .\end{cases}
$$

Let us suppose that $(i i)$ is true, so we infer that

$$
l<\lambda^{\star}(\alpha), \forall \alpha>1
$$

Let us choose a fixed $\alpha_{1}>1$, if $w$ is the "small" solution of

$$
\left\{\begin{array}{l}
\Delta h+l(1+h)^{\alpha_{1}}=0 \text { in } B_{1} \\
h>0 \text { in } B_{1} \\
h=0 \text { on } \partial B_{1}
\end{array}\right.
$$

Using the inequality (1), we infer that

$$
u_{\lambda^{\star}(\alpha)} \geq w, \forall \alpha \geq \alpha_{1}
$$

where $u_{\lambda^{\star}(\alpha)}$ is the unique solution of

$$
\left\{\begin{array}{l}
\Delta u+\lambda^{\star}(\alpha)(1+u)^{\alpha}=0 \text { in } B_{1} \\
u>0 \text { in } B_{1} \\
u=0 \text { on } \partial B_{1}
\end{array}\right.
$$

Let $G$ be the Green's function of the first kind for $-\Delta$, on $B_{1}$ (see [4],

p. 19), we have the following identity

$$
u_{\lambda^{\star}(\alpha)}(x)=\lambda^{\star}(\alpha) \int_{B_{1}} G(x, y)\left(1+u_{\lambda^{\star}(\alpha)}(y)\right)^{\alpha} d y, \forall x \in \overline{B_{1}} .
$$

Let $\epsilon$ and $\delta$ be two positive fixed constants, such that $0<\epsilon<1-\delta<1$, consider the set

$$
B(\epsilon, \delta)=\left\{x \in B_{1}, \epsilon<\|x\|<1-\delta\right\}
$$

The set $B(\epsilon, \delta)$ is not empty. Let us choose $x_{0} \in B(\epsilon, \delta)$, so we infer that

$$
\frac{1}{\lambda^{\star}(\alpha)}=\frac{\int_{B_{1}} G\left(x_{0}, y\right)\left(1+u_{\lambda^{\star}(\alpha)}(y)\right)^{\alpha} d y}{u_{\lambda^{\star}(\alpha)}\left(x_{0}\right)} \geq \frac{\int_{B(\epsilon, \delta)} G\left(x_{0}, y\right)\left(1+u_{\lambda^{\star}(\alpha)}(y)\right)^{\alpha} d y}{u_{\lambda^{\star}(\alpha)}\left(x_{0}\right)} .
$$


As we have (see Lemma 1)

$$
u_{\lambda^{\star}(\alpha)}\left(x_{0}\right)=u_{\lambda^{\star}(\alpha)}\left(\left\|x_{0}\right\|\right) \leq \epsilon^{\star}\left(B_{\left\|x_{0}\right\|}, \alpha\right) \leq\left\|x_{0}\right\|^{\frac{2}{1-\alpha}} \epsilon^{\star}\left(B_{1}, \alpha\right) .
$$

We get that

$$
\frac{1}{\lambda^{\star}(\alpha)} \geq \frac{\int_{B(\epsilon, \delta)} G\left(x_{0}, y\right)\left(1+u_{\lambda^{\star}(\alpha)}(y)\right)^{\alpha} d y}{\left\|x_{0}\right\|^{\frac{2}{1-\alpha}} \epsilon^{\star}\left(B_{1}, \alpha\right)} .
$$

We deduce that

$$
\frac{\epsilon^{\star}\left(B_{1}, \alpha\right)}{\lambda^{\star}(\alpha)} \geq \inf _{z \in B(\epsilon, \delta)}\left(1+u_{\lambda^{\star}(\alpha)}(z)\right)^{\alpha} \frac{\int_{B(\epsilon, \delta)} G\left(x_{0}, y\right) d y}{\left\|x_{0}\right\|^{\frac{2}{1-\alpha}}} .
$$

We use Lemma 1 again, to verify that

$$
\frac{1}{\left(\lambda^{\star}(\alpha)\right)^{1-\frac{1}{\alpha-1}}} \geq \inf _{z \in B(\epsilon, \delta)}\left(1+u_{\lambda^{\star}(\alpha)}(z)\right)^{\alpha} \frac{\int_{B(\epsilon, \delta)} G\left(x_{0}, y\right) d y}{\left\|x_{0}\right\|^{\frac{2}{1-\alpha}}} .
$$

But for every $\alpha \geq \alpha_{1}$, we have

$$
\inf _{z \in B(\epsilon, \delta)}\left(1+u_{\lambda^{\star}(\alpha)}(z)\right)^{\alpha} \geq \inf _{z \in B(\epsilon, \delta)}(1+w(z))^{\alpha} .
$$

As $w$ is radially symmetrical and decreasing, we have

$$
\inf _{z \in B(\epsilon, \delta)}(1+w(z))^{\alpha}=(1+w(1-\delta))^{\alpha} .
$$

The Maximum Principle guarantees that, $w(1-\delta)>0$. So we get that

$$
\lim _{\alpha \rightarrow \infty}(1+w(1-\delta))^{\alpha}=+\infty
$$

as

$$
\lim _{\alpha \rightarrow \infty} \frac{1}{\left(\lambda^{\star}(\alpha)\right)^{1-\frac{1}{\alpha-1}}}=\frac{1}{l}>0
$$

and

$$
\lim _{\alpha \rightarrow \infty}\left\|x_{0}\right\|^{\frac{2}{1-\alpha}}=1,
$$

we get a contradiction from (2). This completes the proof of the first part of Proposition 4.

As we have

$$
u_{\lambda^{\star}(\alpha)}(x)=\lambda^{\star}(\alpha) \int_{B_{1}} G(x, y)\left(1+u_{\lambda^{\star}(\alpha)}(y)\right)^{\alpha} d y, \forall x \in \overline{B_{1}} .
$$

Let us choose $x=O$, using the fact that $u_{\lambda^{\star}(\alpha)}$ is radially symmetrical and has its maximum at the origin, we infer that

$$
\frac{1}{\lambda^{\star}(\alpha)} \leq \frac{\left(1+u_{\lambda^{\star}(\alpha)}(0)\right)^{\alpha}}{u_{\lambda^{\star}(\alpha)}(0)} \int_{B_{1}} G(O, y) d y .
$$

As $\lambda^{\star}(\alpha) \rightarrow 0$, when $\alpha \rightarrow \infty$, we infer that necessarily,

$$
u_{\lambda^{\star}(\alpha)}(0) \rightarrow \infty, \text { as } \alpha \rightarrow \infty
$$


Proposition 6 Let us suppose that there exists $b_{0}>0$ and $\left.r_{0} \in\right] 0,1[$, such that

$$
u_{\lambda^{\star}(\alpha)}\left(r_{0}\right) \leq b_{0}, \forall \alpha>1
$$

Then there exists a positive constant $c$, such that

$$
\lim _{\alpha \rightarrow \infty} u_{\lambda^{\star}(\alpha)}(r)=u_{0}(r) \equiv \frac{c}{n-2}\left(r^{2-n}-1\right), \forall r \in\left[r_{0}, 1\right] .
$$

Proof

As $u_{\lambda^{\star}(\alpha)}$ is a decreasing function, we infer that

$$
u_{\lambda^{\star}(\alpha)}(r) \leq b_{0}, \forall \alpha>1, \forall r \in\left[r_{0}, 1\right]
$$

So we can use the $L^{p}$-estimates and Morrey Theorem (see [4]), to show that

$$
u_{\lambda^{\star}(\alpha)} \rightarrow u_{0} \text { in } C^{1, \delta}\left(\overline{B\left(r_{0}, 1\right)}\right), \forall 0<\delta<1 .
$$

As, $\lambda^{\star}(\alpha) \rightarrow 0$, when $\alpha \rightarrow \infty$, we deduce that

$$
\Delta u_{0}(x)=0 \text { in } B\left(r_{0}, 1\right) .
$$

As the function $u_{0}$ is radially symmetrical, we get that

$$
r^{n-1}\left(r^{n-1} u_{0}^{\prime}(r)\right)^{\prime}=0, \forall r \in\left[r_{0}, 1[.\right.
$$

So we infer that, there exists a positive constant $c$, such that

$$
u_{0}(r)=\frac{c}{n-2}\left(r^{2-n}-1\right), \forall r \in\left[r_{0}, 1\right]
$$

This ends the Proof.

We conjecture that

$$
\left.\left.\lim _{\alpha \rightarrow \infty} u_{\lambda^{\star}(\alpha)}(r)=\frac{c}{n-2}\left(r^{2-n}-1\right), \forall r \in\right] 0,1\right] .
$$

Let us give an immediate corollary of Proposition 5. Let $a$ be a positive regular function on $\overline{B_{1}}$, then,

Corollary 1 The problem

$$
\left\{\begin{array}{l}
\Delta u+a(x)(1+u(x))^{\alpha}=0 \text { in } B_{1} \\
u>0 \text { in } B_{1} \\
u=0 \text { on } \partial B_{1}
\end{array}\right.
$$

doesn't admit a solution, for $\alpha$ "great" enough. 


\section{Some Numerical Computations}

Let us choose $n=3$ and consider the "small" solution $u_{\lambda}$ (when it exists) of the following problem

$$
\left\{\begin{array}{l}
\Delta u+\lambda(1+u)^{2}=0 \text { in } B_{1}, \\
u>0 \text { in } B_{1}, \\
u=0 \text { on } \partial B_{1},
\end{array}\right.
$$

As we have already mentioned in Section 1 , we can put $v_{\lambda}(x)=\lambda u_{\lambda}(x)+\lambda$, so we obtain

$$
\left\{\begin{array}{l}
\Delta v_{\lambda}+v_{\lambda}^{2}=0 \text { in } B_{1}, \\
v_{\lambda}>0 \text { in } B_{1}, \\
v_{\lambda}=\lambda \text { on } \partial B_{1} .
\end{array}\right.
$$

As $v_{\lambda}$ is radially symmetrical, we rewrite the first equation as follows,

$$
r\left(v_{\lambda} "(r)+v_{\lambda}^{2}(r)\right)=-2 v_{\lambda}^{\prime}(r), \forall 0 \leq r<1
$$

If

$$
v_{\lambda}(r)=\Sigma_{j=0}^{\infty} c_{j} r^{j}
$$

we obtain

$$
c_{j}=-\frac{1}{(j+1) j} \Sigma_{k=0}^{j-2} c_{k} c_{j-2-k}, \forall j \geq 2 .
$$

As $v_{\lambda}^{\prime}(0)=0$, we infer that

$$
\begin{gathered}
c_{2 j+1}=0, \forall j \geq 0, \text { and } \\
v_{\lambda}(r)=c_{0}-\Sigma_{j=1}^{\infty}\left(\frac{1}{(2 j+1)(2 j)} \Sigma_{k=0}^{j-1} c_{2 k} c_{2 j-2-2 k}\right) r^{2 j} .
\end{gathered}
$$

As we have

$$
\lambda=v_{\lambda}(1)=c_{0}-\Sigma_{k=1}^{\infty} \frac{1}{(2 j+1)(2 j)} \Sigma_{k=0}^{j-1} c_{2 k} c_{2 j-2-2 k},
$$

let us compute the values of the application (putting $c_{0}=c$ )

$$
f: c \longrightarrow c-\Sigma_{k=1}^{\infty} \frac{1}{(2 j+1)(2 j)} \Sigma_{k=0}^{j-1} c_{2 k} c_{2 j-2-2 k} .
$$

We have written a short Maple Procedure (see the Appendix) which computes and plots this curve. The following curve is the output. 


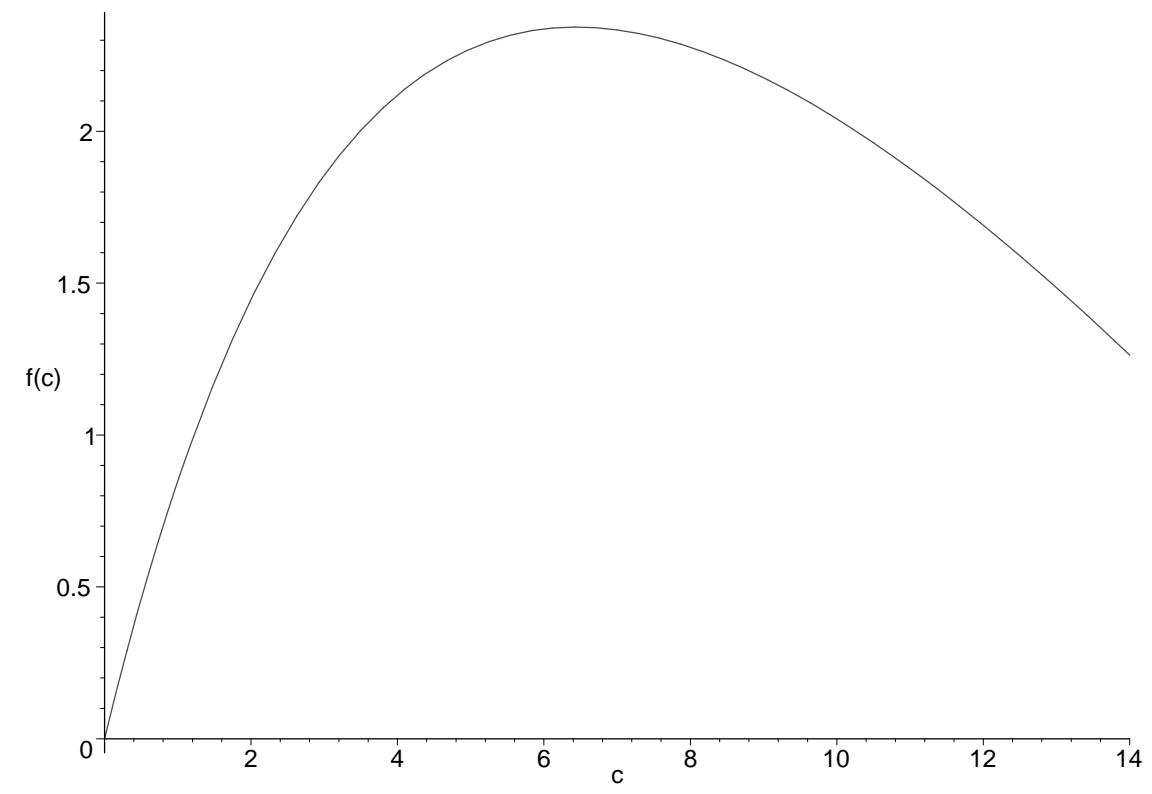

As it appears from the curve, one can compute $\lambda^{\star}(2) \approx 2.343255111$, with $c=c_{0} \approx 6.4407$.

When $c \approx 15$, the computations of $f\left(c_{0}\right)$ "cease" to converge. Is it a loss of analycity or an unstability effect?

\section{ACKNOWLEDGMENTS}

This work was done within the framework of the Associate Scheme of the Abdus Salam International Centre for Theoretical Physics, Trieste, Italy. Financial support from the Swedish International Development Cooperation Agency is acknowledged.

A mes chères enfants Kénizé et Maïna. 
APPENDIX

interface(quiet $=$ true);

$\mathrm{g}:=\operatorname{proc}(\mathrm{a}:$ :nonneg $)$

local M,i,j,k;

$\mathrm{M}[0]:=\mathrm{a} ;$

for i from 1 to 201 do

$\mathrm{M}[\mathrm{i}]:=-\left(1 /\left(2^{*} \mathrm{i}^{*}\left(2^{*} \mathrm{i}+1\right)\right)\right){ }^{*} \operatorname{sum}\left(\mathrm{M}[\mathrm{j}]{ }^{*} \mathrm{M}[\mathrm{i}-1-\mathrm{j}], \mathrm{j}=0 . . \mathrm{i}-1\right)$

od; evalf(sum( $(\mathrm{M}[\mathrm{k}], \mathrm{k}=0 . .201))$;

end:

plotsetup (ps,plotoutput='test.ps' $)$;

with(plots);

$\operatorname{plot}\left(\mathrm{g}, 0 . .14\right.$, labels $\left.=\left[{ }^{6} \mathrm{c}^{6}, \mathrm{f}(\mathrm{c})^{6}\right]\right)$; 


\section{References}

[1] H. BREZIS and L. NIRENBERG, Positive Solutions of Nonlinear Elliptic Equations Involving Critical Sobolev Exponent, Comm. Pure Appl. Math. 36 (1983), 437-477.

[2] M. G. CRANDALL and P.H. RABINOWITZ, Some Continuation and Variational Methods for Positive Solutions of Nonlinear Elliptic Eigenvalue Problems, Arch. Rational Mech. Anal. 58 (1975), 207-218.

[3] B.GIDAS, W.-M. NI and L.NIRENBERG, Symmetry and Related Properties via the Maximum Principle, Comm. Math. Phys. 68 (1979), 209-243.

[4] D. GILBARG and N.S. TRUDINGER, "Elliptic Partial Differential Equations of Second Order," Springer Verlag, 1983.

[5] O.A.-I.-B.ISSELKOU: A Critical Value for the Boundary Datum of a Dirichlet's Problem, Funkcialaj Ekvacioj 41 (1998), 207-214.

[6] O.A.-I.-B.ISSELKOU, Donnéee au Bord Critique pour un Problème de Dirichlet, Revue URED No 8 et 9 (1999), Dakar.

[7] O.A.-I.-B.ISSELKOU, CRITICAL BOUNDARY CONSTANTS AND POHOZAEV IDENTITY, Preprint ICTP, No IC/98/218, Trieste, 1998.

[8] D.D.JOSEPH and T.S.LUNDGREN, Quasilinear Dirichlet Problems Driven by Positive Sources, Arch. Rational Mech. Anal. 49 (1973), 241-269.

[9] H.B. KELLER, Some Positone Problems Suggested by the Nonlinear Heat Generation, in "Bifurcation Theory and Nonlinear Eigenvalues Problems," W.A. BENJAMIN, INC., 1969. 